PROCEEDINGS OF THE

AMERICAN MATHEMATICAL SOCIETY

Volume 126, Number 8, August 1998, Pages 2285-2289

S 0002-9939(98)04435-9

\title{
ON THE MERGELYAN APPROXIMATION PROPERTY ON PSEUDOCONVEX DOMAINS IN $\mathbb{C}^{n}$
}

\author{
SANGHYUN CHO
}

(Communicated by Steven R. Bell)

\begin{abstract}
Let $\Omega$ be a smoothly bounded pseudoconvex domain of finite type in $\mathbb{C}^{n}$. We prove the Mergelyan approximation property in various topologies on $\Omega$ when the estimates for $\bar{\partial}$-equation are known in the corresponding topologies.
\end{abstract}

\section{INTRODUCTION}

Let $\Omega$ be a bounded domain in $\mathbb{C}^{n}$ and let $H(\Omega)$ denote the functions holomorphic on $\Omega$. We say $\Omega$ has the Mergelyan property if every $f \in H(\Omega) \cap C(\bar{\Omega})$ can be approximated uniformly on $\bar{\Omega}$ by functions in $H(\bar{\Omega})$, where $H(\bar{\Omega})$ denotes the functions holomorphic in a neighborhood of $\bar{\Omega}$.

For $n>1$, Henkin [9], Kerzman [10] and Lieb [11] proved the Mergelyan property on strongly pseudoconvex domains in $\mathbb{C}^{n}$. The key technical tools needed to prove the Mergelyan property were:

(1) existence of a Stein neighborhood basis for $\bar{\Omega}$, and

(2) Lipschitz estimates and their stability for $\bar{\partial}$ on this Stein neighborhood basis.

Notice that the author has constructed a Stein neighborhood basis of $\bar{\Omega}$ when $\Omega$ is a smoothly bounded pseudoconvex domain of finite type in $\mathbb{C}^{n}[3]$.

Let $\Lambda^{\alpha}(\Omega)$ denote the usual Lipschitz space of order $\alpha \geq 0$ with norm \|\|$_{\Lambda^{\alpha}(\Omega)}$ and let $L_{k}^{p}(\Omega)$ denote the space of functions on $\Omega$ that are in $L^{p}(\Omega)$ along with all their derivatives up to order $k$, with norm denoted by \|\|$_{L_{k}^{p}(\Omega)}$. Lipschitz estimates for $\bar{\partial}$ on pseudoconvex domains in $\mathbb{C}^{n}$ are known for some special kinds of domains; that is, smoothly bounded pseudoconvex domains of finite type in $\mathbb{C}^{2}[2]$, [7], and smoothly bounded pseudoconvex domain $\Omega$ of finite type in $\mathbb{C}^{n}$ such that the Leviform of $b \Omega$ is diagonizable [8], etc. However, it is difficult and sometimes tedious to prove the stability of the estimates for $\bar{\partial}$ even though the estimates are known $[6],[12]$.

In this paper, we will present a new method to prove the Mergelyan property in various topologies. That is, if $\bar{\Omega}$ has a Stein neighborhood basis with the estimates for $\bar{\partial}$ in $\Lambda^{\alpha}(\Omega), \alpha \geq 0$, or in $L_{k}^{p}(\Omega), 1<p<\infty, k \geq 0$, we will prove the Mergelyan

Received by the editors January 7, 1997.

1991 Mathematics Subject Classification. Primary 32F20, 32H40.

Key words and phrases. Mergelyan property, Lipschitz continuity, finite 1-type.

The author was partially supported by Basic Sci. Res. fund BSRI-97-1411, and by GARCKOSEF, 1997.

(C)1998 American Mathematical Society 
property in the corresponding topologies. Here we are not assuming the stability of the estimates for $\bar{\partial}$ in the corresponding spaces. Nevertheless, we will use the known result of the stability of estimates for $\bar{\partial}$ in $L_{k}^{2}(\Omega)$ spaces [4].

We will state and prove our results only on smoothly bounded pseudoconvex domains of finite type in $\mathbb{C}^{2}$. The same (or similar) results hold for the domains of finite type in $\mathbb{C}^{n}$ with estimates for $\bar{\partial}$ in the corresponding spaces.

Theorem 1. Let $\Omega$ be a smoothly bounded pseudoconvex domain of finite type in $\mathbb{C}^{2}$. Assume $f \in H(\Omega) \cap L_{k}^{p}(\Omega)$, where $1<p<\infty$ and $k$ is a non-negative integer. Then there is a sequence $\left\{g_{n}\right\} \subset H(\bar{\Omega})$ such that

$$
\lim _{n \rightarrow \infty}\left\|g_{n}-f\right\|_{L_{k}^{p}(\Omega)}=0 .
$$

Let $\mathbb{N}$ denote the set of natural numbers.

Theorem 2. Let $\Omega$ be as in Theorem 1 and assume $f \in H(\Omega) \cap \Lambda^{\alpha}(\Omega)$. Then for each $\alpha^{\prime}<\alpha\left(\alpha^{\prime}=\alpha\right.$ if $\left.\alpha \in\{0\} \cup \mathbb{N}\right)$ arbitrarily given, there is a sequence $\left\{g_{n}\right\} \subset H(\bar{\Omega})$ such that

$$
\lim _{n \rightarrow \infty}\left\|g_{n}-f\right\|_{\Lambda^{\alpha^{\prime}}(\Omega)}=0
$$

Remark 3. In [5], the author proved that every $f \in H(\Omega) \cap L_{k}^{2}(\Omega)$ can be approximated by functions in $H(\bar{\Omega})$ in the $L_{k}^{2}(\Omega)$ topologies, $k \geq 0$, when $\Omega$ is a smoothly bounded pseudoconvex domain of finite type in $\mathbb{C}^{n}$. When $n=2$, Cho and others [6] also proved the Mergelyan property in Lipschitz spaces $\Lambda^{\alpha}(\Omega), 0 \leq \alpha<1 / m$, for $f \in H(\Omega) \cap \Lambda^{\alpha}(\Omega)$. Here $m$ is the type of $b \Omega$. Both of these results depend on the solvability and stability of the estimates for $\bar{\partial}$ in the corresponding topologies [4], [6].

The key ingredients needed to prove Theorem 1 and Theorem 2 are the $L_{k}^{p}(\Omega)$ and Lipschitz estimates for $\bar{\partial}$ on $\Omega \Subset \mathbb{C}^{2}[2],[7],[8]$, and the smooth bumping theorem for pseudoconvex domains of finite type in $\mathbb{C}^{n}[2],[3],[4]$.

\section{Approximation By Smooth Functions}

In this section, we prove that any holomorphic function in $L_{k}^{p}(\Omega)$ or $\Lambda^{\alpha}(\Omega)$ can be approximated by smooth functions on $\bar{\Omega}$ in appropriate topologies.

Let $U_{j}, j=0,1, \ldots, N$, be a finite collection of open sets with the following properties:

(a) $\bar{\Omega} \subset \bigcup_{j=0}^{N} U_{j}$.

(b) $U_{0} \Subset \Omega$.

(c) On each $U_{j}, j=1,2, \ldots, N$, there are holomorphic coordinates $z_{1}^{j}$, $z_{2}^{j}$ with the property that $\partial r / \partial x_{2}^{j}>0$, where $z_{2}^{j}=x_{2}^{j}+i y_{2}^{j}$.

Let $\zeta_{j}, j=0,1, \ldots, N$, be a partition of unity subordinate to the covering $\left\{U_{j}\right\}$. For all sufficiently small $\delta>0$, and for a given function $f \in H(\Omega)$, let $f_{\delta}$ be given by

$$
f_{\delta}(z)=\zeta_{0}(z) f(z)+\sum_{j=1}^{N} \zeta_{j}(z) f\left(z_{1}^{j}, z_{2}^{j}-\delta\right) .
$$

Observe that $f_{\delta} \in C^{\infty}(\bar{\Omega})$. 
Proposition 4. Suppose that $f \in H(\Omega) \cap L_{k}^{p}(\Omega), 1<p<\infty, k \geq 0$. Then $\left\|f_{\delta}-f\right\|_{L_{k}^{p}(\Omega)} \longrightarrow 0$ and $\left\|\bar{\partial} f_{\delta}\right\|_{L_{k}^{p}(\Omega)} \longrightarrow 0$ as $\delta \longrightarrow 0$.

Proof. Note that $D^{\alpha}\left(f-f_{\delta}\right) \in L^{p}(\Omega),|\alpha| \leq k$. So $\left\|f_{\delta}-f\right\|_{L_{k}^{p}(\Omega)}$ converges to zero as $\delta$ does, by the Lebesgue dominated convergence theorem. Also note that

$$
D^{\alpha}\left(\bar{\partial} f_{\delta}\right)=D^{\alpha}\left(\bar{\partial}\left(f_{\delta}-f\right)\right)=D^{\alpha}\left(\sum_{j=1}^{N}\left(\bar{\partial} \zeta_{j}\right)\left(f\left(z_{1}^{j}, z_{2}^{j}-\delta\right)-f\left(z_{1}^{j}, z_{2}^{j}\right)\right)\right) .
$$

So $\left\|\bar{\partial} f_{\delta}\right\|_{L_{k}^{p}(\Omega)} \longrightarrow 0$ as $\delta \longrightarrow 0$ by the same reasoning.

The functions $f_{\delta} \in C^{\infty}(\bar{\Omega})$ defined in (1) also approximate $f$ in the $\Lambda^{\alpha}(\Omega)$ topology:

Proposition 5. Suppose that $f \in H(\Omega) \cap \Lambda^{\alpha}(\Omega)$. Then for each $\alpha^{\prime}<\alpha\left(\alpha^{\prime}=\alpha\right.$ if $\alpha \in\{0\} \cup \mathbb{N}),\left\|f_{\delta}-f\right\|_{\Lambda^{\alpha^{\prime}}(\Omega)}$ and $\left\|\bar{\partial} f_{\delta}\right\|_{\Lambda^{\alpha^{\prime}}(\Omega)}$ converge to zero as $\delta$ does.

Proof. It is clear that the conclution holds if $\alpha \in\{0\} \cup \mathbb{N}$. Without loss of generality, we may assume that $0<\alpha^{\prime}<\alpha<1$. If $x, y$ satisfy $\delta \leq|x-y|$, we use the Lipschitz continuity condition on $\left(f_{\delta}-f\right)(x)$ and $\left(f_{\delta}-f\right)(y)$, and if $\delta>|x-y|$, we use the same condition on $f(x)-f(y)$ and $f_{\delta}(x)-f_{\delta}(y)$. In both cases, we will get $\left\|f_{\delta}-f\right\|_{\Lambda^{\alpha}(\Omega)} \lesssim \delta^{\alpha-\alpha^{\prime}}$. Since $\alpha^{\prime}<\alpha$, this proves that $\left\|f_{\delta}-f\right\|_{\Lambda^{\alpha}(\Omega)}$ converges to zero as $\delta$ does. Similarly, we can prove that $\left\|\bar{\partial} f_{\delta}\right\|_{\Lambda^{\alpha}(\Omega)}$ converges to zero as $\delta$ does.

Remark 6. If $\alpha \notin \mathbb{N} \cup\{0\}$, the functions $f_{\delta}$ defined in (1) does not converge to $f$ in the $\Lambda^{\alpha}(\Omega)$ norm in general.

Definition 7. Let $\Omega \subset \mathbb{C}^{n}$ be a smoothly bounded pseudoconvex domain with $C^{\infty}$ defining function $r$. By a smooth bumping family of $\Omega$ we mean a family of smoothly bounded pseudoconvex domains $\left\{\Omega_{\tau}\right\}_{0 \leq \tau \leq 1}$ satisfying the following properties:

(1) $\Omega_{0}=\Omega$,

(2) $\Omega_{\tau_{1}} \Subset \Omega_{\tau_{2}}$ if $\tau_{1}<\tau_{2}$,

(3) $\left\{b \Omega_{\tau}\right\}_{0 \leq \tau \leq 1}$ is a $C^{\infty}$ family of real hypersurfaces in $\mathbb{C}^{n}$,

(4) the boundary defining functions $r_{\tau}$ of $\Omega_{\tau}$ vary smoothly with respect to $\tau$, and $r_{\tau} \rightarrow r$ as $t \rightarrow 0$ in the $C^{\infty}$ topology.

In [3], the author constructed a smooth bumping family of $\Omega$ if $b \Omega$ is of finite type.

For the final remark of this section, we state the following theorem which gives the stability of $L^{2}$-estimates for $\bar{\partial}$ on $\Omega[4]$.

Theorem 8. Let $\left\{\Omega_{\tau}\right\}_{0 \leq \tau \leq 1}$ be a smooth bumping family of pseudoconvex domains in $\mathbb{C}^{n}$. Then for each $m$ there exists a constant $C_{m}$, independent of $\tau$, such that

$$
\left\|f^{\tau}\right\|_{m} \leq C_{m}\left\|\square_{\tau} f^{\tau}\right\|_{m},
$$

for all $f^{\tau} \in \operatorname{Dom}\left(\square_{\tau}\right) \cap C^{\infty}\left(\bar{\Omega}_{\tau}\right)$ with $f^{\tau} \perp H^{0,1}\left(\bar{\Omega}_{\tau}\right)$. Here $\square_{\tau}$ denotes the complex Laplacian on $\Omega_{\tau}$. 


\section{Approximation By holomorphic functions}

Let $\Omega$ be a smoothly bounded pseudoconvex domain of finite type in $\mathbb{C}^{2}$, and let $N$ be the Neumann operator associated with the $\bar{\partial}$-Neumann problem. Then the main results in [2], [7] show that

$$
\bar{\partial}^{*} N: L_{k}^{p}(\Omega) \longrightarrow L_{k}^{p}(\Omega), \quad 1<p<\infty, \quad k \geq 0,
$$

and

$$
\bar{\partial}^{*} N: \Lambda^{\alpha}(\Omega) \longrightarrow \Lambda^{\alpha}(\Omega), \quad \alpha \geq 0,
$$

are bounded operators on the corresponding spaces.

Now let us prove Theorem 1 and Theorem 2. Here we will only present a proof of Theorem 2. The proof of Theorem 1 follows the same (or similar) lines. Let us state Theorem 2 again:

Theorem 2. Let $\Omega$ be a smoothly bounded pseudoconvex domain of finite type in $\mathbb{C}^{2}$. Assume $f \in H(\Omega) \cap \Lambda^{\alpha}(\Omega)$. Then for each $\alpha^{\prime}<\alpha\left(\alpha^{\prime}=\alpha\right.$ if $\left.\alpha \in\{0\} \cup \mathbb{N}\right)$ arbitrarily given, there is a sequence $\left\{g_{n}\right\} \subset H(\bar{\Omega})$ such that

$$
\lim _{n \rightarrow \infty}\left\|g_{n}-f\right\|_{\Lambda^{\alpha^{\prime}}(\Omega)}=0
$$

Proof. Let $f_{\delta}$ be defined by (1). By Proposition $5, f_{\delta} \in C^{\infty}(\bar{\Omega})$ converge to $f$ in the $\Lambda^{\alpha^{\prime}}(\Omega)$ topology as $\delta$ goes to zero. For each fixed $\delta>0$, let us solve $\bar{\partial} u_{\delta}=\bar{\partial} f_{\delta}$ on $\Omega$. Then by Catlin's global regularity theorem for the $\bar{\partial}$-equation on pseudoconvex domains of finite type in $\mathbb{C}^{n}[1]$, it follows that $u_{\delta} \in C^{\infty}(\bar{\Omega})$. Also, by (3), $u_{\delta}$ satisfies

$$
\left\|u_{\delta}\right\|_{\Lambda^{\alpha^{\prime}(\Omega)}} \leq C_{\alpha}\left\|\bar{\partial} f_{\delta}\right\|_{\Lambda^{\alpha^{\prime}(\Omega)}},
$$

where $C_{\alpha}$ does not depend on $\delta$. Since $\left\|\bar{\partial} f_{\delta}\right\|_{\Lambda^{\alpha^{\prime}}(\Omega)}$ converges to zero as $\delta$ does, it follows that $\left\|u_{\delta}\right\|_{\Lambda^{\alpha^{\prime}(\Omega)}} \rightarrow 0$ as $\delta \rightarrow 0$. Set

$$
h_{\delta}=f_{\delta}-u_{\delta} .
$$

Then $h_{\delta} \in H(\Omega) \cap C^{\infty}(\bar{\Omega})$ and

$$
\lim _{\delta \rightarrow 0}\left\|h_{\delta}-f\right\|_{\Lambda^{\alpha^{\prime}}(\Omega)}=0 .
$$

Let $\left\{\Omega_{\tau}\right\}$ be a smooth pseudoconvex bumping family of $\Omega$. We extend $h_{\delta}$ to $\Omega_{\tau}$ and set it equal to $h_{\delta}^{\tau}$ on $\Omega_{\tau}$. Notice that $\bar{\partial} h_{\delta}^{\tau} \equiv 0$ on $\Omega$, and $\bar{\partial} h_{\delta}^{\tau} \in C^{\infty}(\bar{\Omega})$. Hence $\bar{\partial} h_{\delta}^{\tau}$ vanishes to order infinity on $b \Omega$ as $\tau \rightarrow 0$. Set $k=[\alpha]+4$, where $[\alpha]$ denotes the greatest integer less than or equal to $\alpha$. Let us solve the following $\bar{\partial}$-equation in $L_{k}^{2}(\Omega)$ spaces (with weighted estimates of $\bar{\partial}$ ):

$$
\bar{\partial} p_{\delta}^{\tau}=\bar{\partial} h_{\delta}^{\tau} \text { on } \Omega_{\tau} .
$$

From Theorem 8 (stability of $L_{k}^{2}\left(\Omega_{\tau}\right)$-estimates of $\bar{\partial}$-equation), it follows that

$$
\left\|p_{\delta}^{\tau}\right\|_{L_{k}^{2}\left(\Omega_{\tau}\right)} \leq C_{\alpha}\left\|\bar{\partial} h_{\delta}^{\tau}\right\|_{L_{k}^{2}\left(\Omega_{\tau}\right)},
$$

where $C_{\alpha}$ does not depend on $\tau$. Set

$$
g_{\delta}^{\tau}=p_{\delta}^{\tau}-h_{\delta}^{\tau} \in H\left(\Omega_{\tau}\right) .
$$

Then (4), (5) and the Sobolev embedding theorem imply that

$$
\left\|g_{\delta}^{\tau}-f\right\|_{\Lambda^{\alpha^{\prime}}(\Omega)} \longrightarrow 0 \text { as } \delta, \tau \rightarrow 0 .
$$

This proves Theorem 2 . 


\section{REFERENCES}

1. Catlin, D.W., Global regularity of the $\bar{\partial}-$ Neumann problem, Proc. Sympos. Pure Math. 41 (1984), 39-49. MR 85j:32033

2. Chang, D.C., Nagel, A. and Stein, E., Estimates for the $\bar{\partial}-$ Neumann problem in pseudoconvex domains of finite type in $\mathbb{C}^{2}$, Acta Math. 169 (1992), 153-228. MR 93k:32025

3. Cho, S., Extension of complex structures on weakly pseudoconvex compact complex manifolds with boundary, Math. Z. 211 (1992), 105-119. MR 94h:32030

4. H. R. Cho, S. Cho, and K. H. Shon, Stability of the estimates for $\bar{\partial}$-equation on compact pseudoconvex complex manifolds, Kyushu J. Math. 48-1 (1994), 19-34. MR 95k:32017

5. Cho, H.R. and Cho, S., Holomorphic approximation on compact pseudoconvex complex manifolds, J. of Geom. Anal. (to appear).

6. Cho, S., Ahn, H. and Kim, S., Stability of Hölder estimates for $\bar{\partial}$ on pseudoconvex domains of finite type in $\mathbb{C}^{2}$, Nagoya Math. J. (to appear).

7. Fefferman, C. and Kohn, J.J., Hölder estimates on domains of complex dimension two and on three dimensional CR manifolds, Adv. Math. 69 (1988), 233-303. MR 89g:32027

8. Fefferman, C., Kohn, J.J. and Machedon, M., Hölder estimates on CR manifolds with diagonalizable Levi form, Adv. Math. 84 (1990), 1-90. MR 92a:32019

9. G. M. Henkin, Integral representations of functions which are holomorphic in strictly pseudoconvex regions, and some applications, Math. Sb. 78 (1969), 611-632; , Engl. Transl.: Math. USSR. Sb. 7 (1969), 597-616. MR 40:2902

10. N. Kerzman, Hölder and $L^{p}$ estimates for solutions of $\bar{\partial} u=f$ in strongly pseudoconvex domains, Comm. Pure Appl. Math. 24 (1971), 301-379. MR 43:7658

11. I. Lieb, Ein Approximationssatz auf streng pseudokonvexen Gebieten, Math. Ann. 184 (1969), 56-60. MR 41:7146

12. Range, R.M., Integral kernels and Hölder estimates for $\bar{\partial}$ on pseudoconvex domains of finite type in $\mathbb{C}^{2}$, Math.Ann. 288 (1990), 63-74. MR 91j:32018

Department of Mathematics, Sogang University, C.P.O. Box 1142, Seoul 121-742, Korea

E-mail address: shcho@ccs.sogang.ac.kr 